\title{
A plastic waveguide receiver in 40nm CMOS with on-chip bondwire antenna
}

\author{
Maarten Tytgat and Patrick Reynaert \\ KU Leuven ESAT-MICAS \\ Kasteelpark Arenberg 10 \\ B-3001 Leuven, Belgium \\ Email: patrick.reynaert@esat.kuleuven.be
}

\begin{abstract}
This paper presents the design and measurements of a $40 \mathrm{~nm}$ CMOS BPSK and multilevel ASK receiver for wired connections through a plastic waveguide, operating at $87 \mathrm{GHz}$. In a measurement setup containing the receiver chip with bondwire dipole antenna and a long piece of polypropylene waveguide with a rectangular cross-section of $2.2 \mathrm{~mm}$ by $0.9 \mathrm{~mm}$, a maximum datarate of $9 \mathrm{Gbit} / \mathrm{s}$ over a distance of $60 \mathrm{~cm}$ and $2.5 \mathrm{Gbit} / \mathrm{s}$ over a distance of $9 \mathrm{~m}$ is measured, both with a bit error rate of less than $10^{-12}$ and a PRBS length of $2^{7}-1$. The chip consumes 50 $\mathrm{mW}$ of DC power from a $0.9 \mathrm{~V}$ supply. The total chip area is 2.1 $\mathrm{mm}^{2}$, of which $52 \%$ is occupied by the antenna and reflector.
\end{abstract}

\section{INTRODUCTION}

Continuous scaling in CMOS has led to the use of higher carrier frequencies in wireless data communication systems. A trend towards millimeter wave integrated circuits is apparent from recent publications [1]-[4]. A direct consequence of this increase in frequency is the size reduction of passive circuit elements such as transmission lines, transformers and antennas, which are now enabled to be integrated on a single chip. In this work, the small wavelength is exploited to make a direct interface between a chip and a flexible dielectric waveguide with a cross-section in the order of millimeters. This way, a wired data connection can be set up through a low loss channel, which allows to transmit gigabits of data per second over a distance of several meters.

A plastic waveguide link combines the best from the worlds of optical fibers and wireless millimeter wave communication. It is similar to optical fiber as it uses a low-loss dielectric channel. But just as in a wireless millimeter wave link, the use of more advanced modulation schemes, such as BPSK, QPSK, FSK and QAM is possible. Thanks to the confinement of the EM-wave in the dielectric, the free space path loss is eliminated, resulting in a high SNR at the receiver without the need for highly directional antennas. Additional advantages over optical fiber are the use of cheap plastics such as polypropylene, polystyrene or polyethylene and the use of standard CMOS without the need for electrical-to-optical or optical-to-electrical converters.

A plastic waveguide link consists of a transmitter and a receiver chip on each end of a dielectric waveguide. Antennas couple the millimeter wave signal from the chip to the waveguide and vice versa. In [3], these antennas are implemented off-chip on a seperate substrate. In this work, the antennas are implemented using bondwires so they can be integrated with the chip in the same package.

This paper presents the design of a $40 \mathrm{~nm}$ CMOS receiver chip with bondwire antenna and measurements of a complete plastic waveguide link with a transmitter built from measurement equipment. Section II discusses the different components of the system: the CMOS chip, the bondwire antenna and the plastic waveguide. Section III describes measurements to characterize the chip and the performance of the complete link. Bit error rates measurements up to $9 \mathrm{~m}$ distance are presented.

\section{SYSTEM COMPONENTS}

\section{A. CMOS receiver chip}

The receiver consists of an LNA, an injection locked VCO, a double balanced mixer and a series of baseband amplifiers. The schematic is shown in figure 1.

The LNA is a cascade of three identical transformer-coupled differential common source stages. Neutralization capacitance is added for reverse isolation and differential mode stability. Extra resistors are added in the bias lines to improve common mode stability.

As in [3], the signal from the LNA is split. One part is sent to the injection path, which contains an additional buffer stage. The other part goes to the bottom part of a double balanced Gilbert mixer. The neutralization in the bottom pair of the mixer eases the impedance matching between the output of the LNA and the RF input of the mixer.

The VCO is designed to oscillate at $90 \mathrm{GHz}$, based on [4], and to lock onto the incoming signal to cover dynamic frequency fluctuations in the transmitter VCO. The control voltage can be adjusted to compensate for design errors that result in static frequency deviations.

The baseband amplifiers are the same as in [1]. The last stage is designed to drive the off-chip $50 \Omega$ load.

\section{B. Antenna}

The antenna is the interface between the plastic waveguide and the receiver circuit. A dipole antenna is made by two bondwires, because this results in lower loss than a dipole fabricated in the top metal [5]. A plane in the top metal acts as a reflector and shield underneath the antenna. The antenna and shield were simulated in HFSS, together with the plastic waveguide. The antenna is optimized for maximum power transfer from the waveguide to the chip at $90 \mathrm{GHz}$. The total 


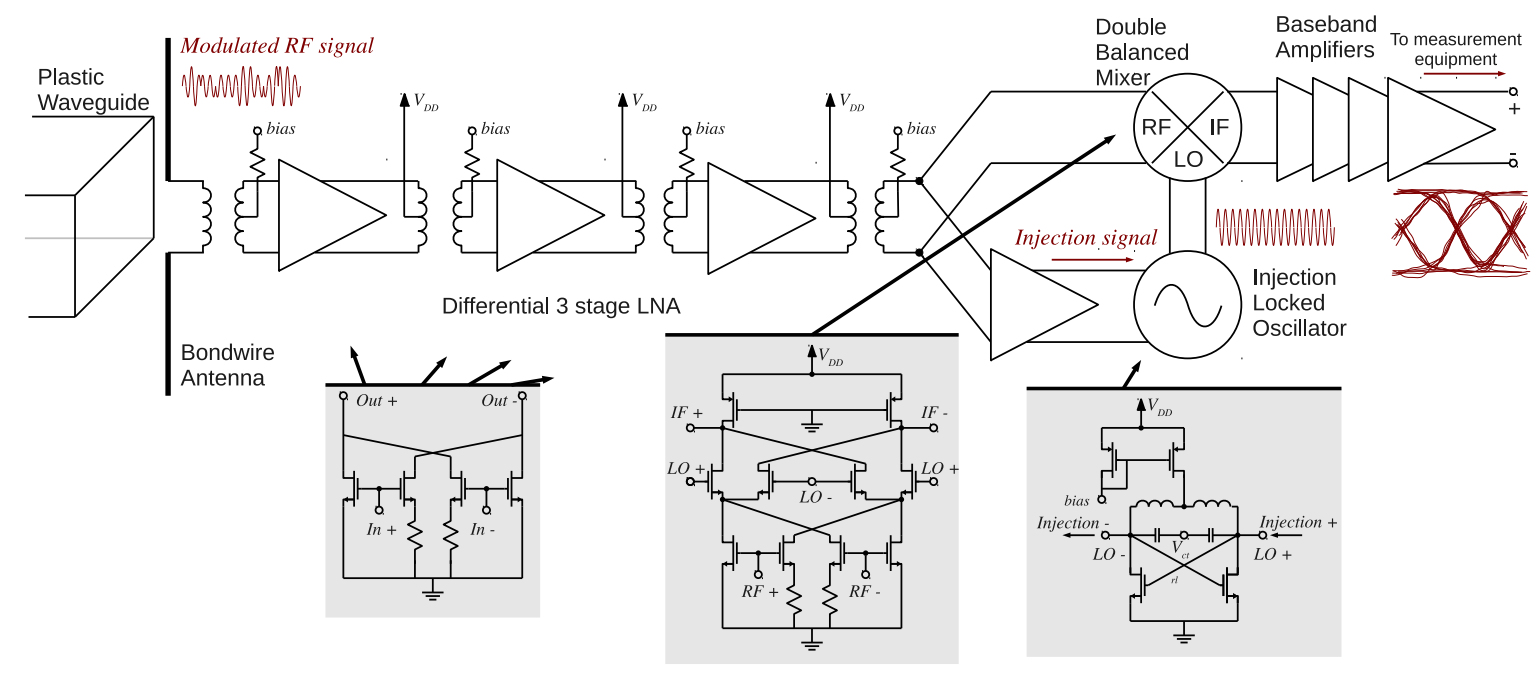

Fig. 1. Presented chip

chip area occupied by the antenna and the shield is $1.1 \mathrm{~mm}^{2}$, which is $52 \%$ of the total chip area. The area underneath the shield can however be used to place digital circuits or analog baseband circuits.

\section{Plastic Waveguide}

Plastic waveguides can be made in different forms. In [6], an analysis is given of circular, elliptical and rectangular dielectric waveguides. For this work, a rectangular crosssection is preferred because it interfaces more easily with metal waveguides and the polarization of the electromagnetic wave is unambiguous.

According to [7], two kinds of modes can exist in a rectangular plastic waveguide: the $E_{n m}^{x}$ modes, with their electric field mainly polarized in the $x$-direction, and the $E_{n m}^{y}$ modes, with their electric field mainly polarized in the $y$-direction. The $n$, $m$ represent the mode order, or the number of extrema of the field components in respectively the $x$ and the $y$ direction. The $z$ direction lies along the length of the waveguide.

In this work, only the $E_{n m}^{y}$ modes are present because of the polarization of the antenna, or in the case of the measurement setup described later on, because the plastic waveguide is inserted in a metal waveguide, which defines the polarization. Which modes can propagate, depends on the frequency and the dimensions and the dielectric constant $\epsilon_{r}$ of the waveguide. In [7], the propagation constant for plastic waveguides is approximated with closed formulas. According to these formulas, the propagation constant $k_{z}$ for a plastic waveguide in polypropylene with a cross-section of $2.2 \mathrm{~mm}$ by $0.9 \mathrm{~mm}$ is calculated for the different modes as shown in figure 2. It can be seen that monomode propagation is possible for frequencies between 78 and $102 \mathrm{GHz}$, which fits the carrier frequency of $87 \mathrm{GHz}$.

The material is chosen based on the dielectric loss. This is quantified by the loss tangent, $\tan \delta$. According to [6] page 173 , at $30 \mathrm{GHz}$, the dielectric constant and the loss tangent for polypropylene are $\epsilon_{r}=2.261$ and $\tan \delta=0.5 \times 10^{-3}$.

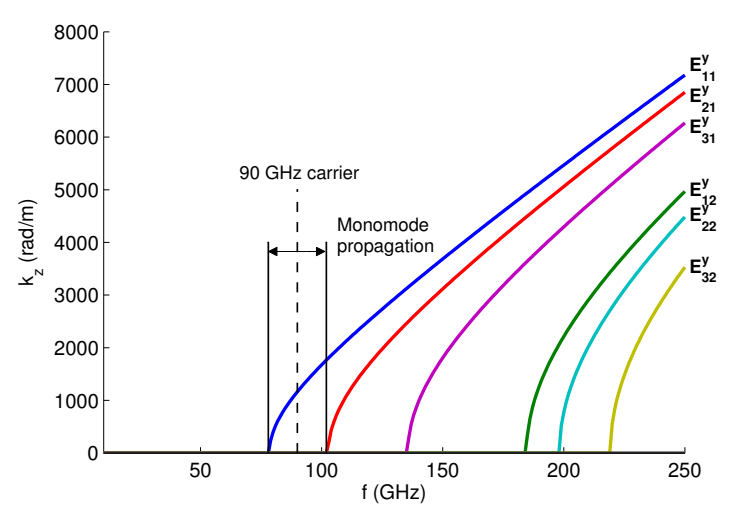

Fig. 2. Calculated propagation constant according to [7] for polypropylene with a cross-section of $2.2 \mathrm{~mm}$ by $0.9 \mathrm{~mm}$

A loss between 1 and $3 \mathrm{~dB} / \mathrm{m}$, depending on the frequency is estimated by measuring $S_{21}$ between 90 and $140 \mathrm{GHz}$ with a network analyzer and subtracting reflection losses.

\section{MeAsurements}

The chip is fabricated in a $40 \mathrm{~nm}$ standard CMOS process. It is glued and wire bonded to an FR-4 substrate. A chip photograph is shown in figure 3 .

One end of a $2.2 \mathrm{~mm}$ by $0.9 \mathrm{~mm}$ polypropylene waveguide is inserted into a metal waveguide to apply the RF signal. The other end is brought close to the bondwire antenna on the chip. This is shown in figure 4.

The RF signal is generated with an Agilent E8257D PSG combined with an OML S12MS source module. For measurements without modulated signals, the plastic waveguide is connected directly to the output of the source module. When modulated signals are needed, the setup in figure 8 is used.

\section{A. Chip characterization}

The total power consumption is $50 \mathrm{~mW}$ from a $0.9 \mathrm{~V}$ supply, which includes the buffers to drive the off-chip 50 


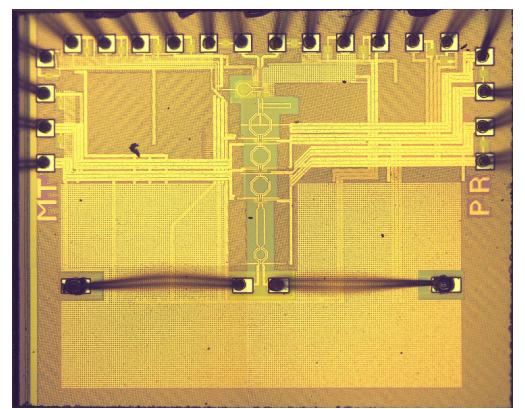

Fig. 3. Micrograph of the chip with the on-chip bondwire antenne at the bottom

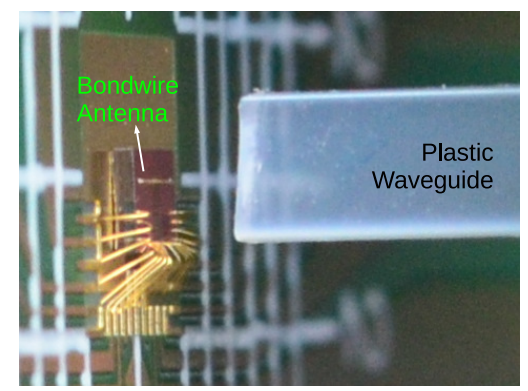

Fig. 4. Chip wire-bonded to PCB with end of plastic waveguide

$\Omega$ impedance of the measurement equipment.

The total area, including antenna, reflector and bondpads, is $2.1 \mathrm{~mm}^{2}$. The active area is only $0.21 \mathrm{~mm}^{2}$.

The baseband frequency response (figure 5) of the chip is measured by applying a $87 \mathrm{GHz}$ RF signal that is amplitude modulated with a sine wave of which the frequency is increased. The on-chip mixer down-converts this signal to baseband. The resulting sine wave is measured with a spectrum analyzer. The baseband bandwidth is partly limited by the bondwires and the PCB trace.

The tuning range of the VCO is measured using a spectrum analyzer with an external mixer to pick up the weak signal from the VCO directly with a horn antenna. The tuning range for a control voltage of $-1 \mathrm{~V}$ to $1 \mathrm{~V}$ is $89.5 \mathrm{GHz}$ to $83.1 \mathrm{GHz}$, as can be seen in figure 6 .

The locking behaviour of the ILO can be examined by applying an RF signal and looking at the IF response on a spectrum analyzer. As long as the ILO is locked to the RF input signal, there's only a DC voltage at the output, but as

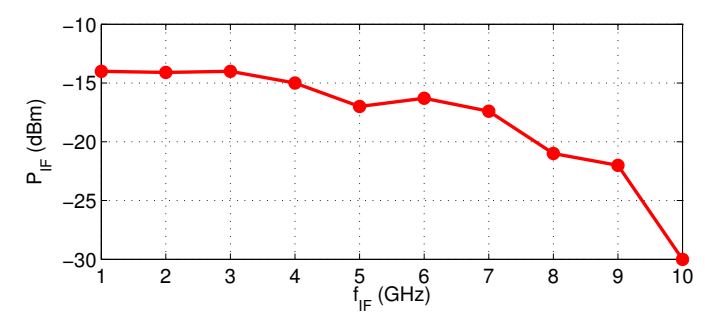

Fig. 5. Measured baseband frequency response

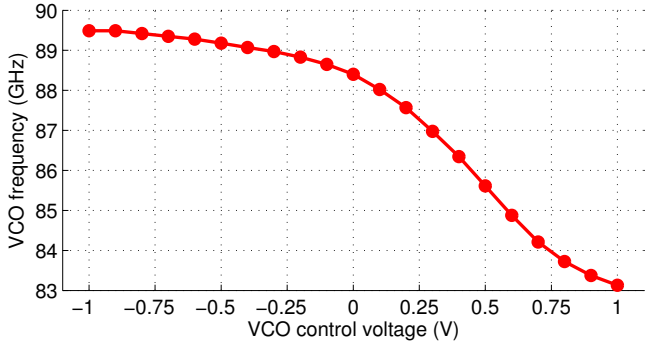

Fig. 6. Tuning range of the VCO

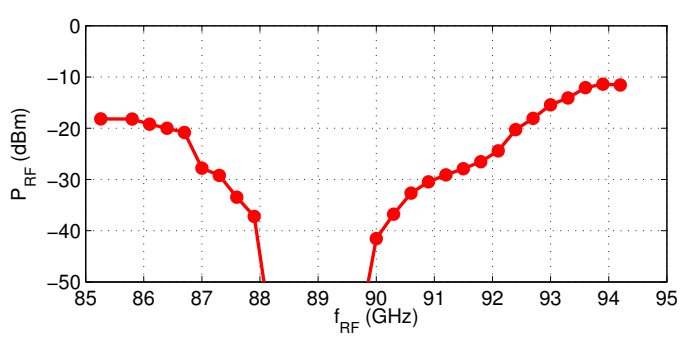

Fig. 7. Locking range of the $\mathrm{VCO}, V_{c t r l}=0 \mathrm{~V}$

soon as lock is lost, the difference frequency between the LO and the RF signal is visible on the spectrum analyzer. Whether or not the ILO will lock to the RF at a certain frequency depends on the RF power. For each RF frequency, the input power is lowered untill lock is lost. This power is marked on figure 7. On the $y$-axis, the power coming out of the plastic waveguide is indicated. From the graph, it is seen that lock is most easily achieved at the free running frequency of the VCO.

\section{B. Modulated signals and complete link measurements}

For the generation of Gbps modulated signals at $90 \mathrm{GHz}$, the setup of figure 8 is used. The millimeter wave signal from the source module is amplified to drive the LO port of a 80-90 GHz mixer. The baseband signal comes either from a Tektronix AWG7122B arbitrary waveform generator (AWG) or an Agilent 81250 parallel bit error rate tester (ParBERT). An attenuator is used to set the desired amplitude level. The power that comes out of the metal waveguide is measured with an Erickson PM4 power meter. Unless stated otherwise, this level is set to $0 \mathrm{dBm}$, a value that is certainly feasible to be generated with modern CMOS processes [2], [3].

To demonstrate multilevel ASK demodulation, the AWG was used to generate a four level ASK signal. An eye diagram of a $4 \mathrm{Gbit} / \mathrm{s}$ signal, demodulated by the chip, is shown in figure 9. For BPSK signals, the receiver locks on the LO feedthrough that occurs in the transmitter of the measurement setup. The ParBERT was used to test bit error rates. For all BER measurements, a PRBS length of $2^{7}-1$ is used. Different lengths of plastic waveguide are tested to determine the influence of the channel, namely power loss and dispersion, on the signal quality. The results can be found in figure 10 . The carrier frequency is $87 \mathrm{GHz}$. When a confidence level of 


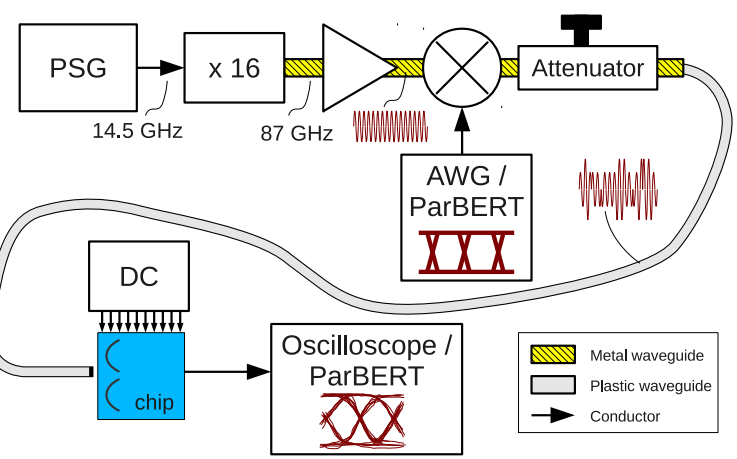

Fig. 8. Measurement setup for data rate testing. A flexible plastic waveguide connects the modulated signal generator to the receiver chip

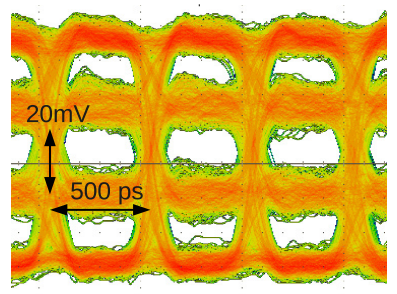

Fig. 9. Eye diagram of a four level ASK signal of $4 \mathrm{Gbit} / \mathrm{s}$, demodulated by the chip

$95 \%$ is reached for a bit error rate less than BER $<10^{-12}$ [8], the measurement is stopped to avoid very long measurement times.

The maximum datarate measured for a BER $<10^{-12}$ is 9 Gbps, over a distance of $60 \mathrm{~cm}$. The energy efficiency of the receiver is then $5.6 \mathrm{pJ} / \mathrm{bit}$. For a distance of $9 \mathrm{~m}$, the maximum bitrate measured for BER $<10^{-12}$ is $2.5 \mathrm{Gbps}$. The energy efficiency of the receiver is then $20 \mathrm{pJ} / \mathrm{bit}$. At $9 \mathrm{~m}$, the loss in the waveguide is measured to be $23 \mathrm{~dB}$.

\section{CONCLUSION}

This paper presents the design and measurement of a 87 $\mathrm{GHz}$ injection locked BPSK and multilevel ASK receiver

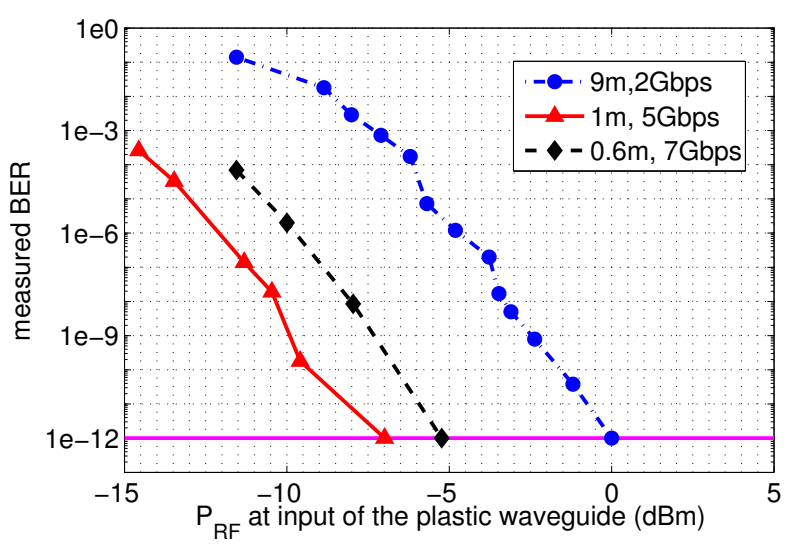

Fig. 10. Measured BER versus input power to the polypropylene waveguide for different data rates and waveguide lengths at $87 \mathrm{GHz}$ carrier frequency
TABLE I

PERFORMANCE SUMMARY AND COMPARISON TO OTHER WORK

\begin{tabular}{|l|c|c|c|}
\hline & $\begin{array}{c}{[3] 57 \mathrm{GHz}} \\
\mathrm{Rx}\end{array}$ & $\begin{array}{c}{[3] 80 \mathrm{GHz}} \\
\mathrm{Rx}\end{array}$ & This work \\
\hline Carrier Frequency & $57 \mathrm{GHz}$ & $80 \mathrm{GHz}$ & $87 \mathrm{GHz}$ \\
\hline Tx power & $0 \mathrm{dBm}$ & $0 \mathrm{dBm}$ & $0 \mathrm{dBm}$ \\
\hline Highest bitrate & $15 \mathrm{Gbps}$ & $12.5 \mathrm{Gbps}$ & $9 \mathrm{Gbps}$ \\
at distance & $0.1 \mathrm{~m}$ & $0.12 \mathrm{~m}$ & $0.6 \mathrm{~m}$ \\
Bitrate $\times$ distance & $1.5 \mathrm{Gbps} . \mathrm{m}$ & $1.5 \mathrm{Gbps} . \mathrm{m}$ & $5.4 \mathrm{Gbps} . \mathrm{m}$ \\
\hline Longest distance & $1 \mathrm{~m}$ & & $9 \mathrm{~m}$ \\
Maximum bitrate & $10 \mathrm{Gbps}$ & & $2.5 \mathrm{Gbps}$ \\
Bitrate $\times$ distance & $10 \mathrm{Gbps} . \mathrm{m}$ & & $22.5 \mathrm{Gbps} . \mathrm{m}$ \\
\hline DC power & $42 \mathrm{~mW}$ & $45 \mathrm{~mW}$ & $50 \mathrm{~mW}$ \\
\hline Supply & $1.1 \mathrm{~V}$ & $1.1 \mathrm{~V}$ & $0.9 \mathrm{~V}$ \\
\hline Modulation & ASK & ASK & BPSK \& \\
& & & multilevel ASK \\
\hline Active area & $0.14 \mathrm{~mm}{ }^{2}$ & $0.14 \mathrm{~mm}{ }^{2}$ & $0.21 \mathrm{~mm}{ }^{2}$ \\
\hline Antenna & Quasi-Yagi & Quasi-Yagi & Dipole on-chip \\
& on PCB & on PCB & bondwire \\
\hline Technology & $40 \mathrm{~nm}$ & $40 \mathrm{~nm}$ & $40 \mathrm{~nm}$ \\
& CMOS & CMOS & CMOS \\
\hline
\end{tabular}

with on-chip bondwire antenna. A plastic waveguide link was demonstrated with bitrates up to 9 Gbps for a distance of 60 $\mathrm{cm}$ and $2.5 \mathrm{Gbps}$ for a distance of $9 \mathrm{~m}$. Table I summarizes the performance and compares this work to the two receivers in [3]. They propose a full-duplex interconnect using two links at different frequencies in the same plastic waveguide. This work reports the highest bitrate - distance product.

\section{ACKNOWLEDGMENT}

Thanks to Flanders' PlasticVision for the production of the plastic waveguide.

This research is partly supported by the ERC Advanced Grant 227680 (DARWIN).

\section{REFERENCES}

[1] M. Tytgat, M. Steyaert, and P. Reynaert, "A 186 to $212 \mathrm{GHz}$ downconverter in 90nm CMOS," Journal of Infrared, Millimeter, and Terahertz Waves, vol. 33, no. 11, pp. 1085-1103, 2012.

[2] N. Deferm and P. Reynaert, "A $120 \mathrm{GHz} 10 \mathrm{~Gb} / \mathrm{s}$ phase-modulating transmitter in 65nm LP CMOS," in Solid-State Circuits Conference Digest of Technical Papers (ISSCC), 2011 IEEE International, feb. 2011, pp. 290 -292 .

[3] S. Fukuda, Y. Hino, S. Ohashi, T. Takeda, H. Yamagishi, S. Shinke, K. Komori, M. Uno, Y. Akiyama, K. Kawasaki, and A. Hajimiri, "A $12.5+12.5 \mathrm{~Gb} / \mathrm{s}$ full-duplex plastic waveguide interconnect," Solid-State Circuits, IEEE Journal of, vol. 46, no. 12, pp. 3113 -3125, dec. 2011.

[4] W. Volkaerts, M. Steyaert, and P. Reynaert, "118GHz fundamental VCO with $7.8 \%$ tuning range in 65nm CMOS," in Radio Frequency Integrated Circuits Symposium (RFIC), 2011 IEEE, june 2011, pp. 1 -4.

[5] U. Johannsen, A. Smolders, J. Leiss, and U. Gollor, "Bond-wires: Readily available integrated millimeter-wave antennas," in Microwave Conference (EuMC), 2012 42nd European, 2012, pp. 197-200.

[6] C. Yeh and F. Shimabukuro, The Essence of Dielectric Waveguides. Springer, 2008.

[7] E. Marcatili, "Dielectric rectangular waveguide and directional coupler for integrated optics," in Bell System Technical Journal, v48: i7 September 1969, sep 1969, pp. $2071-2102$.

[8] Maxim, Statistical Confidence Levels for Estimating Error Probability, 2010. [Online]. Available: http://notesapplication.abcelectronique.com/003/3-5321.pdf 